M. Umehara

Nagoya Math. J.

Vol. 134 (1994), 75-89

\title{
6-VERTEX THEOREM FOR CLOSED PLANAR CURVE WHICH BOUNDS AN IMMERSED SURFACE WITH NON-ZERO GENUS
}

\author{
MASAAKI UMEHARA \\ Dedicated to Professor Hideki Ozeki on his sixtieth birthday
}

\section{Introduction}

A vertex of a planar curve $\gamma$ of class $\mathscr{C}^{2}$ is a point which attains a local maximum or minimum of its curvature function. By the definition, the number of vertices are even whenever it is finite. As a generalization of famous four vertex theorem, Pinkall [P] showed that a closed curve $\gamma$ has at least 4 vertices if it bounds an immersed surface, and he conjectured that $\gamma$ has at least $4 g+2$ vertices when the surface has genus $g$.

As a counter example of the conjecture, recently, Cairns, Özdemir and Tjaden [COT] pointed out that for each genus $g \geq 1$, there is a closed planar curve with 6 vertices that bounds an immersed surface of genus $g$. In addition, they conjectured that the lowest number of the vertices of a closed planer curve is 6 if it bounds an immersed surface other than the disc. In this note, we show their conjecture is true:

THEOREM. Let $\gamma$ be a closed planar curve of class $\mathscr{C}^{2}$ which bounds an immersed surface. Suppose that $\gamma$ has exactly 4 vertices. Then it bounds only the disc.

The author is very grateful to Osamu Kobayashi for his valuable suggestions.

\section{Preliminaries}

In this section, we review the properties of vertices. Let $\gamma=\gamma(s): \mathbf{R} \rightarrow \mathbf{R}^{2}$ be a $l$-periodic curve of class $\mathscr{C}^{2}$, parametrized by arc length. We mean such an periodic curve with the parameter by the terminology "closed planar curve". We

Received April 12, 1993. 
take the normal vector field $n=n(s)$ of $\gamma$ such that the frame $\{\dot{\gamma}, n\}$ is positively oriented. The curvature function $k=k(s)$ is defined by the Frenet-Serret equation $\ddot{\gamma}=k n$.

The curve $\gamma$ is said to have a maximal (resp. minimal) vertex at $s=s_{0}$, if $R\left(s_{0}\right)$ is local maximum (resp. minimum) of the curvature function. Since the number of the maximal and the minimal vertices are the same, the number of vertices are even unless it is infinite.

A geometric meaning of vertex is the following: (cf. [J]) The curve $\gamma$ has a maximal (resp. minimal) vertex at $s=s_{0}$ if and only if there exists a positive number $\varepsilon>0$ such that the image $\left.\gamma\right|_{\left[s_{0}-\varepsilon, s_{0}+\varepsilon\right]}$ is contained in the right (resp. left) hand side of the osculating circle at $s=s_{0}$. An orientation preserving Möbius transformation $T$ is written in the form

$$
T(z)=\frac{a z+b}{c z+d} \quad(a d-b c=1, a, b, c, d \in \mathbf{C}),
$$

where we identify the plane $\left(\mathbf{R}^{2} ; x, y\right)$ with $\mathbf{C}$ and set $z=x+i y$. Since $T$ maps an oriented circle to a circle with the same orientation, the following lemma is obvious:

Lemma 1.1. Let $\gamma$ be a planar curve. Then any orientation preserving Möbius transformation maps maximal (resp. minimal) vertices of $\gamma$ to the same one of $T \circ \gamma$.

Next we consider self-intersections of the curve $\gamma$.

DeFinition 1.2. The planar curve $\gamma$ is said to have a shell on the open interval $I=(a, b)$, if $\gamma(a)=\gamma(b)(0 \leq a<b \leq l)$ and $\gamma$ has no self-intersection on the interval $I$.

If $\gamma$ has shells on intervals $I_{1}, I_{2}, \ldots, I_{n}$ respectively, then these shells are called independent if

$$
\pi\left(I_{i}\right) \cap \pi\left(I_{j}\right)=\phi \quad(1 \leq i<j \leq n)
$$

holds, where $\pi: \mathbf{R} \rightarrow \mathbf{R} / l \mathbf{Z}$ is the canonical projection. The following lemma is obtained from the fact: If $\gamma(a)=\gamma(b) \quad(0 \leq a<b \leq l)$, then there exists an open subinterval $I$ of $(a, b)$ such that the restriction $\left.\gamma\right|_{I}$ has no self-intersection.

Lemma 1.3. Let $\gamma$ be a closed planar curve which has a self-intersection. Then $\gamma$ has at least two independent shells. 
The each shell $\left.\gamma\right|_{I}$ of $\gamma$ bounds a domain $D$ which is called the interior of the shell. The internal angle $\mu$ to $D$ at the intersection point is called the internal angle of the shell $\left.\gamma\right|_{I}$. (Fig. 1) We remark that each shell admits two possible orientations: If the bounded domain $D$ lies in left-hand (resp. right-hand) side of $\gamma$, the shell is said to have positive (resp. negative) orientation. (Fig. 2)

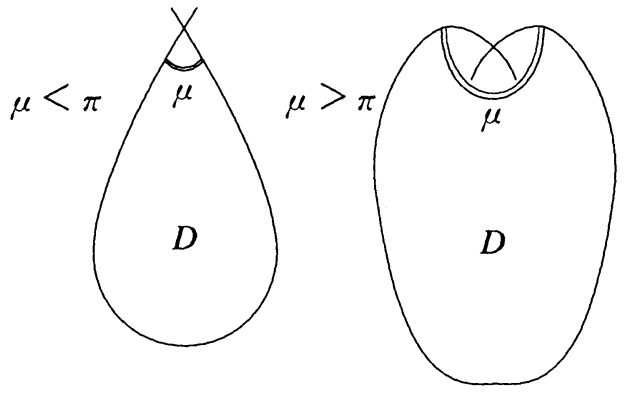

Fig. 1. (Shell)

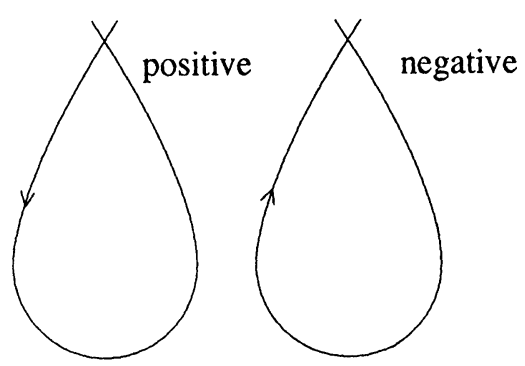

Fig. 2. (Orientations of shell)

The following proposition holds:

Lemma 1.4 (Jackson [J; Lemma 4.3]). Let $\gamma:[a, b] \rightarrow \mathbf{R}^{2}$ be a planar curve such that it has a shell with internal angle $\mu(\leq \pi)$ on the interval $(a, b)$. If the orientation of the shell is positive (resp. negative), there is a maximal (resp. minimal) vertex on $(a, b)$.

By Lemma 1.1, one can reverse a shell by a suitable Möbious transformation. So we rewrite Lemma 1.4 as follows:

LeMma $1.4^{\prime}$ ([J; Lemma 4.3]). Let $\gamma:[a, b] \rightarrow \mathbf{R}^{2}$ be a planar curve such that it has a shell with internal angle $\mu(\geq \pi)$ on the interval $(a, b)$. If the orientation of the shell is positive (resp. negative), there is a minimal (resp. maximal) vertex on $(a, b)$.

COROLlaRy 1.5 (JJ; Cor. 4.3.1]). Let $\gamma:[a, b] \rightarrow \mathbf{R}^{2}$ be a planar curve such that it has a shell with internal angle $\pi$ on the interval $(a, b)$. Then there are at least a pair of maximal and minimal vertices on $(a, b)$.

\section{Positive shell}

We identify $\mathbf{R}^{2} \cup\{\infty\}$ with the unit sphere $S^{2}$ by the stereographic projec- 
tion at the north pole. According to Pinkall [P], we say that a closed planar curve $\gamma$ bounds an immersed surface of genus $g$, if there is a compact orientable surface $M^{2}$ of genus $g$ with connected boundary $\partial M=S^{1}$ and an immersion $x: M^{2} \rightarrow \mathbf{R}^{2}$ $U\{\infty\}$ such that $\gamma=\left.x\right|_{\partial M}$. In this paper, we assume that the local image of the surface $x\left(M^{2}\right)$ lies on the left hand side on the curve $\gamma$.

Now we show the following topological property of such closed curves:

THEOREM 2.1. Let $\gamma: \mathbf{R} \rightarrow \mathbf{R}^{2}$ be a closed planar curve of period $l$, which bounds an immersed surface. Suppose that $\gamma$ has a positive shell of internal angle $(\leq \pi)$ on an interval $(a, b)(0 \leq a<b \leq l)$. Then there exists a negative shell of internal angle $(\leq \pi)$ on some interval $(c, d)(0 \leq c<d \leq l)$ such that the image of $\left.\gamma\right|_{[c, d]}$ is contained in the interior $D$ of the shell on $(a, b)$. In particular, two intervals $(a, b)$ and $(c, d)$ are disjoint.

Remark 2.2. This means the non-existence of solitaty positive shells with internal angle $(\leq \pi)$. The following figure (Fig. 3) demonstrates the theorem.



Fig. 3.

To prove the theorem, we prepare three lemmas:

Lemma 2.3. Let $\gamma$ be a closed planar curve of class $\mathscr{C}^{2}$ which bounds an immersed surface. Suppose that $\gamma$ has a positive shell of internal angle $\mu(\leq \pi)$ on a interval $(a, b)(0 \leq a<b \leq l)$. Then there exists an interval $(c, d)(0 \leq c<d \leq l)$ such that the image of $\left.\gamma\right|_{[c, d]}$ are contained in the interior $D$ of the shell on $(a, b)$.

Proof. We suppose that the interior $D$ does not contain any point on the curve $\gamma$. Let $x: M^{2} \rightarrow \mathbf{R}^{2}$ be an associated immersion such that $\gamma=\left.x\right|_{\partial M}$. By our convention, the local image of the surface $x\left(M^{2}\right)$ lies on the left hand side on the curve $\gamma$ and hence the inverse image $x^{-1}(D)$ is not empty. Let $U$ be a connected 
component of the inverse image $x^{-1}(D)$ whose closure meets $\partial M$. Then the assumption yields that $x(U)=D$ and it can be easily checked that the restriction $\left.x\right|_{U}: U \rightarrow D$ induces a topological finite covering structure. Since $D$ is simply connected, $\left.x\right|_{U}$ is a diffeomorphism.

On the other hand, we set

$$
\gamma_{\varepsilon}(s)=\gamma(s)+\varepsilon \cdot n(s) \quad(s \in \mathbf{R}),
$$

where $n(s)$ is the oriented normal vector field on $\gamma$. Since the statement is purely topological, by a suitable differentiable small perturbation on the shell, we may assume that the internal angle $\mu$ is less than $\pi$. Then for a suitably small $\varepsilon>0$ $\gamma_{\varepsilon}$ has a self-intersection point in $D$. (See Fig. 4.) Since the inverse image of $\gamma_{\varepsilon}$ in $U$ is contained in the boundary of $\varepsilon$-collar of the surface $M^{2}$, this contradicts the fact that $\left.x\right|_{U}$ is a diffeomorphism.

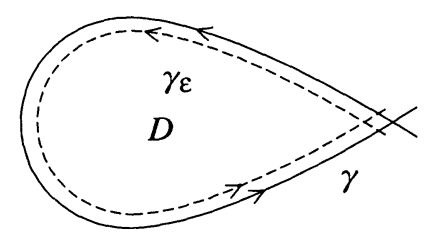

Fig. 4.

Lemma 2.4. Let $\gamma$ be a closed planar curve of class $\mathscr{C}^{2}$ which bounds an immersed surface. Suppose that $\gamma$ has a positive shell of internal angle $\mu(\leq \pi)$ on a interval $(a, b)(0 \leq a<b \leq l)$. Then there exists a shell on some interval $(c, d)$ $(0 \leq c<d \leq l)$ such that the image of $\left.\gamma\right|_{[c, d]}$ is contained in the interior $D$ of the shell on $(a, b)$.

Proof. We suppose that the interior $D$ does not contain any shell on the curve $\gamma$. By Lemma 2.3, we may suppose that there exists an interval $I$ such that the image of $\left.\gamma\right|_{I}$ are contained in $D$. Since the statement is purely topological, by a suitable differentiable small perturbation on the shell, we may assume that the internal angle $\mu$ is less than $\pi$. Since $\mu<\pi$, for a suitably small $\varepsilon_{0}>0, \gamma_{\varepsilon}(0<\varepsilon$ $\left.<\varepsilon_{0}\right)$ defined by (2.1) makes a positive shell of internal angle $(<\pi)$ on some subinterval $\left(a^{\prime}, b^{\prime}\right)$ of $(a, b)$ such that the image of $\left.\gamma_{\varepsilon}\right|_{\left[a^{\prime}, b^{\prime}\right]}$ are contained in $D$. (See Fig. 4.) We denote by $D_{\varepsilon}$ the interior of the new shell $\left.\gamma_{\varepsilon}\right|_{\left(a^{\prime}, b^{\prime}\right)}$.

Let $\left\{I_{\lambda}\right\}_{\lambda \in \Lambda}$ be the splitting of the inverse image $\gamma^{-1}(D)$ as a family of disjoint open subsets of $(0, l)$. Since the length of the closed curve $\gamma$ is finite, only finite 
components, say

$$
\left.\gamma\right|_{I_{1}}, \ldots,\left.\gamma\right|_{I_{n}}
$$

meet the closure of $D_{\varepsilon / 2}$, where $\left\{I_{j}\right\}_{j=1, \ldots, n}$ is a finite subset of $\left\{I_{\lambda}\right\}_{\lambda \in \Lambda}$. Then for each $\left.\gamma\right|_{I_{j}}$, one can patch a simply connected domain smoothly, to avoid the domain $D$. (See Fig. 5.) After these $n$-times surgeries, one can easily see that $D_{\varepsilon}$ does not contain any point on $\gamma_{\varepsilon}$. Though the curve $\gamma_{\varepsilon}$ is in the class $\mathscr{C}^{1}$, the modified argument in the proof of Lemma 2.3 can be applied as follows: We may suppose $\varepsilon<$ $\varepsilon_{0} / 2$. Let $x: M_{\varepsilon}^{2} \rightarrow \mathbf{R}^{2}$ be an associated immersion such that $\gamma=\left.x\right|_{\partial M_{\varepsilon}}$. Let $U_{\varepsilon}$ be a connected component of the inverse image $x^{-1}\left(D_{\varepsilon}\right)$ whose boundary contained in $\partial M_{\varepsilon}$. Then we can conclude that $x\left(U_{\varepsilon}\right)=D_{\varepsilon}$ and it can be easily checked that the restriction $\left.x\right|_{U_{\varepsilon}}: U_{\varepsilon} \rightarrow D_{\varepsilon}$ induces a topological finite covering structure. Since $D_{\varepsilon}$ is simply connected, $\left.x\right|_{U_{\varepsilon}}$ is a diffeomorphism. Since $\gamma_{2 \varepsilon}$ has a self-intersection in $D_{\varepsilon}$, this makes a contradiction.
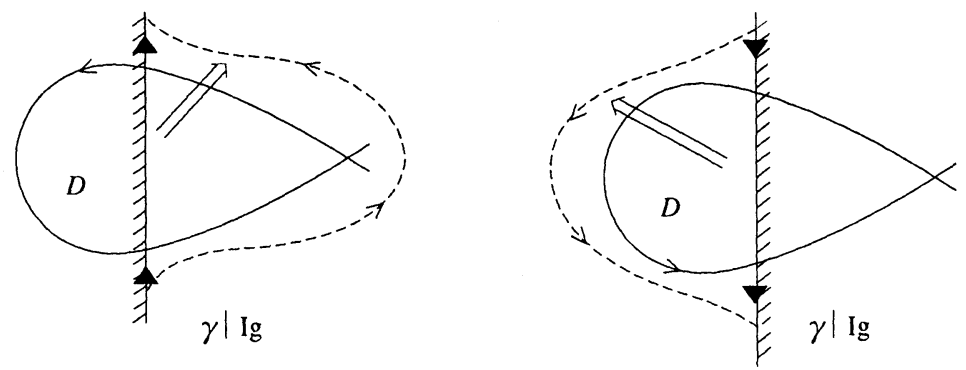

Fig. 5.

LEMma 2.5. Let $\gamma$ be a closed planar curve which has a shell of internal angle $\mu(>\pi)$ on an interval $(a, b)(0 \leq a<b \leq l)$ and $D$ the interior of its shell. Suppose that there exists a number $s_{0}$ such that $\gamma\left(s_{0}\right) \notin \bar{D}$, where $\bar{D}$ is the closure of $D$. Then there exists another shell of internal angle $\mu(\leq \pi)$ on some interval $(c, d)$ $(0 \leq c<d \leq l)$ which is contained in $\bar{D}$.

Proof. Since $\gamma$ is periodic, we may assume that $s_{0}>b$. Then the only two possibilities occur:

(1) There exists an interval $(c, d)\left(a<c<b<d<s_{0}\right)$ such that $\left.\gamma\right|_{(c, d)}$ is a shell of internal angle $(\leq \pi)$ in $\bar{D}$. (See Fig. 6.)

(2) There exists an interval $(c, d)\left(b<c<d<s_{0}\right)$ such that $\left.\gamma\right|_{(c, d)}$ is a shell of internal angle $(\leq \pi)$ in $D$. (See Fig. 7.) 
This proves the lemma.



Fig. 6.

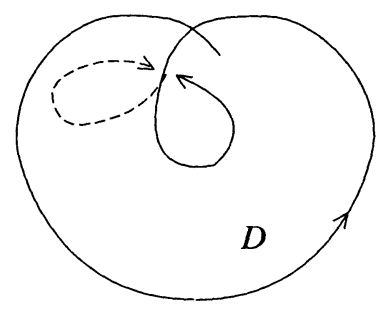

Fig. 7.

Proof of Theorem 2.1. By Lemma 2.4 and Lemma 2.5, $\gamma$ has a shell of internal angle $(\leq \pi)$ on some interval $(c, d)$ which is contained in the original shell. If the new shell is positive, we can apply this argument again. Since $\gamma$ is a immersion with compact image, the number of independent shells is finite. So we find a negative shell of internal angle $(\leq \pi)$ contained in $D$ by repeating the argument.

As an application of Theorem 2.1, we get the followings:

Proposition 2.6. Let $\gamma: \mathbf{R} \rightarrow \mathbf{R}^{2}$ be a closed planar curve of period $l$, which bounds an immersed surface. Suppose that $\gamma$ has at most 4 vertices. Then for any shell on $\gamma$ associated with an interval $(a, b)(0 \leq a<b \leq l)$, there exists $c \in$ $(a, b)$ which attains a minimal vertex.

Proof. When $\gamma$ is a simple closed curve, the proposition is obvious because it has a unique shell $(0, l)$.

So we may assume that $\gamma$ has a self-intersection. By a suitable Möbius transformation, we may also assume that the shell $\left.\gamma\right|_{(a, b)}$ has internal angle $(\mu \leq \pi)$. If the shell has negative orientation, then it has a minimal vertex by Lemma 1.4. So we assume that the shell $\left.\gamma\right|_{(a, b)}$ has positive orientation. Moreover, by Lemma 1.5, we may also assume that the internal angle $\mu$ is less than $\pi$. By Lemma 1.4 , there exists a number $s_{0}\left(a<s_{0}<b\right)$ such that $\gamma\left(s_{0}\right)$ attains maximal vertex. By Theorem 2.1, there is a negative shell $\left.\gamma\right|_{(c, d)}$ of internal angle $(\leq \pi)$ contained in the interior $D$ of the original shell $\left.\gamma\right|_{(a, b)}$. By Lemma 1.4, there exists a number $s_{1}\left(c<s_{1}<d\right)$ such that $\gamma\left(s_{1}\right) \in D$ attains a minimal vertex. Since $\gamma$ is periodic, we may assume that $s_{1}>b$. Since $\gamma$ has at most 4 vertices, either $\left.\gamma\right|_{\left(s_{0}, s_{1}\right)}$ or $\left.\gamma\right|_{\left(s_{1}, s_{0}+l\right)}$ has no vertices. Without loss of generality, we may assume that $\left.\gamma\right|_{\left(s_{0}, s_{1}\right)}$ 
has no vertices. Since any shell has at least one vertex, $\left.r\right|_{\left(s_{0}, s_{1}\right)}$ has no self-intersection. Since $\gamma\left(s_{1}\right) \in D$ and by the assumption $\mu<\pi$, there exist $t_{0} \in$ $(a, b)$ and $t_{1} \in\left(s_{0}, s_{1}\right)$ such that $\gamma\left(t_{0}\right)=\gamma\left(t_{1}\right)$ and $\left.\gamma\right|_{\left(b, t_{1}\right)}$ lies in the complement of $\bar{D}$. Then $\gamma$ has a positive shell of internal angle $(\geq \pi)$ on $\left(t_{0}, t_{1}\right)$. By Lemma $1.4^{\prime}$, there is a minimal vertex on $\left(t_{0}, t_{1}\right)$. Since $\gamma$ has no vertex on $\left(s_{0}, s_{1}\right)$, the minimal vertex lies in a subinterval $\left(t_{0}, s_{0}\right)$ of $(a, b)$. (See Fig. 8.)



Fig. 8.

COROLlary 2.7 (Pinkall [P]). Let $\gamma$ be a closed planar curve which bounds an immersed surface. Then the curve $r$ has at least 4 vertices.

Proof. When $\gamma$ is a simple closed curve, the corollary reduces to the classical 4 -vertex theorem. So we may assume that $\gamma$ has a self-intersection. By Lemma 1.3, $\gamma$ has at least two shells. By Proposition 2.6, the number of minimal vertices of $\gamma$ is greater than or equal to two. This proves the corollary, because the number of maximal vertices is the same as that of the minimal one.

Definition 2.8. A closed planar curve $\gamma: \mathbf{R} \rightarrow \mathbf{R}^{2}$ of period $l$, is called semisimple if there exist numbers $s_{0}$ and $s_{1}\left(s_{0}<s_{1}\right)$ such that the restrictions $\left.\gamma\right|_{\left[s_{0}, s_{1}\right]}$ and $\left.\gamma\right|_{\left[s_{1}, s_{0}+l\right]}$ are embeddings. (See Fig. 9.)

Obviously, a simple closed curve is semisimple. 



Fig. 9. (Examples of semisimple closed curves)

We get the following:

THEOREM 2.9. Let $\gamma: \mathbf{R} \rightarrow \mathbf{R}^{2}$ be a smooth closed planar curve of period $l$, which bounds an immersed surface. Suppose that $\gamma$ has exactly 4 vertices. Then it is semisimple.

Proof. Let $\gamma\left(s_{0}\right)$ and $\gamma\left(s_{1}\right)$ be the minimal vertices of $\gamma$. Then by Proposition 2.6, $\left.\gamma\right|_{\left[s_{0}, s_{1}\right]}$ and $\left.\gamma\right|_{\left[s_{1}, s_{0}+l\right]}$ are embeddings.

\section{Cancellation of intersections}

In this section, we prove the following purely topological assertion:

THEOREM 3.1. Let $\gamma: \mathbf{R} \rightarrow \mathbf{R}^{2}$ be a closed planar curve of period $l$, which bounds an immersed surface. Suppose that $\boldsymbol{\gamma}$ is semisimple. Then it bounds only the disc.

The 6-vertex theorem stated in Introduction immediately follows from Theorem 2.9 and Theorem 3.1 .

Remark 3.2. In the contrast with the semisimple case, for each genus $g$, there is a closed curve which bounds an immersed surface of genus $g$ such that it can be divided by three embedding parts. It is realized by the examples in [COT].

When the curve $\gamma$ is a simple closed curve, the theorem is obvious. So we assume that $\gamma$ is semi-simple, but has a self-intersection. First we observe the fundamental properties of semisimple closed curve: Let $\gamma: \mathbf{R} \rightarrow \mathbf{R}^{2}$ be a closed planar curve of period $l$. We assume that $\gamma$ is semisimple. Then by the definition, 
there exist numbers $s_{0}$ and $s_{1}\left(s_{0}<s_{1}\right)$ such that $\left.\gamma\right|_{\left[s_{0}, s_{1}\right]}$ and $\left.\gamma\right|_{\left[s_{1}, s_{0}+l\right]}$ are embeddings. This division of the closed curve may not be unique. We fix this division and call $\gamma\left(s_{0}\right)$ and $\gamma\left(s_{1}\right)$ connecting points of semisimple closed curve $\gamma$. For the sake of simplicity, we denote $\gamma_{1}=\left.\gamma\right|_{\left[s_{0}, s_{1}\right]}$ and $\gamma_{1}=\left.\gamma\right|_{\left[s_{1}, s_{0}+l\right]}$. The following properties are elementary conclusions of the definition of semisimple closed curve:

(A) After performing a small perturbation, we may assume that all self-intersections of $\gamma$ are transversal. (cf. $[\mathrm{W}]$ )

Hereafter, we assume that any semisimple closed curve has the property (A).

(B) The number of self-intersections is finite and every self-intersection is a double point.

Proof. Suppose that there is a triple point. Then the third image of $\gamma$ does not belong to $\gamma_{1}$ nor $\gamma_{2}$, which yields a contradiction.

(C) The number of independent shells is exactly two.

Proof. Since $\gamma_{1}$ and $\gamma_{2}$ do not have self-intersection, $\gamma$ has at most two independent shells. Thus Lemma 1.3 yields the conclusion.

(D) If $\gamma$ bounds an immersed surface, $\gamma$ has even self-intersections. Moreover, $\gamma$ can be assumed that it has negative shell of internal angle $(<\pi)$.

Proof. By Lemma 5.3 in [KB], $\gamma$ has even self-intersections. By a suitable Möbius transformation, we may assume that $\gamma$ has a shell of internal angle $(<\pi)$. If this shell is positive, we find a negative shell of internal angle $(<\pi)$ by Theorem 2.1 and the property (A).

The following is the key to prove Theorem 3.1.

LEMma 3.3. Let $\gamma$ be a semisimple closed curve, which bounds an immersed surface. Suppose that there are two disjoint closed intervals $[a, b]$ and $[c, d]$ contained in $[0, l]$ such that

(1) $\gamma(a)=\gamma(d)(=P), \gamma(b)=\gamma(c)(=Q)$.

(2) $\left.\gamma\right|_{(a, b)}$ and $\left.\gamma\right|_{(c, d)}$ have no intersection on itself and each other.

(3) The two internal angles of the domain $D$ bounded by $\left.\gamma\right|_{[a, b]}$ and $\left.\gamma\right|_{[c, d]}$ are both less than $\pi$. (Fig. 10) 
Then by an image homotopic deformation of the immersed surface, certain two intersection points of $\gamma$ can be canceled.

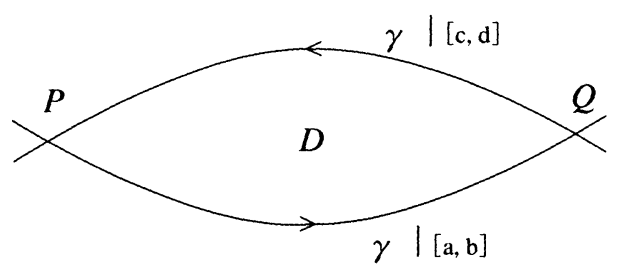

Fig. 10. (a leaf)

Proof. We call such a domain $D$ a leaf. If the leaf $D$ contains no points on $\gamma$, then two intersection $\left.\gamma\right|_{[a, b]}$ and $\left.\gamma\right|_{[c, d]}$ can be canceled, obviously.

Now we assume that $D$ contains points on $\gamma$. By the property $(B)$, the inverse image $\gamma^{-1}(\bar{D})$ is expressed by a finite union of disjoint closed subintervals in $[0, l]$

$$
\gamma^{-1}(\tilde{D})=I_{1} \cup I_{2} \cup \ldots \cup I_{n}
$$

Step 1: First we consider the case that $D$ contains points on $\gamma$ which are not the connecting points. Then each interval $I_{j}=\left[\alpha_{j}, \beta_{j}\right](j=1, \ldots, n)$ is a subinterval of $\left[s_{0}, s_{1}\right]$ or $\left[s_{1}, s_{0}+l\right]$. When $I_{j} \subset\left[s_{0}, s_{1}\right]$ (resp. $I_{j} \subset\left[s_{1}, s_{0}+l\right]$ ), the restriction of the curve $\left.\gamma\right|_{I_{j}}$ belongs $\gamma_{1}$ (resp. $\gamma_{2}$ ) and hence $\left.\gamma\right|_{I_{j}}$ meets $\left.\gamma\right|_{[c, d]}$ (resp. $\left.\left.\gamma\right|_{\{a, b]}\right)$ at $s=\alpha_{j}$ and $s=\beta_{j}$.

So we find a new leaf $D^{\prime}$ bounded by the curves $\left.\gamma\right|_{I_{j}}$ and the restriction of $\left.\gamma\right|_{[c, d]}$ (resp. $\left.\gamma\right|_{[a, b]}$ ), because internal angles are both less than $\pi$. (See Fig. 11.) Replacing $D$ by $D^{\prime}$, we can apply this process. Since the intersection points are finite, repeating this process, we find two disjoint intervals $\left[a^{\prime}, b^{\prime}\right]$ and $\left[c^{\prime}, d^{\prime}\right]$ such that

(i) $\left.\gamma\right|_{\left(a^{\prime}, b^{\prime}\right)}$ and $\left.\gamma\right|_{\left(c^{\prime}, d^{\prime}\right)}$ have no intersection on itself and each other.

(ii) The interior $D^{\prime \prime}$ bounded by $\left.\gamma\right|_{\left[a^{\prime}, b^{\prime}\right]}$ and $\left.\gamma\right|_{\left[c^{\prime}, d^{\prime}\right]}$ contains no points on $\gamma$.

Thus two intersection $\left.\gamma\right|_{\left[a^{\prime}, b^{\prime}\right]}$ and $\left.\gamma\right|_{\left[c^{\prime}, d^{\prime}\right]}$ can be canceled.

Step 2: Next we consider the case that $D$ contains connecting points on $\gamma$. It suffices to show the existence of other two disjoint leaves. Since there are only two connecting points, at least one leaf does not contain them and the discussion 
reduces to Step 1.

Without loss of generality, we may assume that the connecting point $\gamma\left(s_{0}\right)$ is contained in $D$. Then there exist numbers $\alpha$ and $\beta\left(s_{0}<\alpha<a, d<\beta<s_{0}+l\right)$ such that $\left.\gamma\right|_{[\alpha, a]}\left(\right.$ resp. $\left.\left.\gamma\right|_{[d, \beta]}\right)$ meets $\left.\gamma\right|_{[c, d]}\left(\right.$ resp. $\left.\left.\gamma\right|_{[a, b]}\right)$ only at the points $\gamma(\alpha)$ and $\gamma(a)$ (resp. $\gamma(d)$ and $\gamma(\beta)$ ). Obviously, the interior $D_{1}$ (resp. $D_{2}$ ) bounded by $\left.\gamma\right|_{[\alpha, a]}\left(\right.$ resp. $\left.\left.\gamma\right|_{[d, \beta]}\right)$ and a restriction of $\left.\gamma\right|_{[c, a]}$ (resp. $\left.\left.\gamma\right|_{[a, b]}\right)$, has internal angles $(<\pi)$ and so it is a leaf. Moreover, $D$ and $D_{1}$ (resp. $D_{2}$ ) are disjoint. (See Fig. 12.) If $D_{1}$ and $D_{2}$ are also disjoint, we find three disjoint leaves $D, D_{1}$ and $D_{2}$. Next we suppose that $D_{1} \cap D_{2}$ is not empty. Then there exists a number $\xi(d<\xi$ $<\beta)$ such that $\left.\gamma\right|_{[d, \xi]}$ meets $\gamma_{[\alpha, a]}$ only at the points $\gamma(d)$ and $\gamma(\xi)$. Since the domain $D_{3}$ bounded by $\left.\gamma\right|_{[d, \xi]}$ and a restriction of $\left.\gamma\right|_{[\alpha, a]}$, has internal angles $(<\pi)$, it is also a leaf. Obviously, these three leaves $D, D_{1}$ and $D_{3}$ are mutually disjoint. (See Fig. 13.) This proves the lemma.

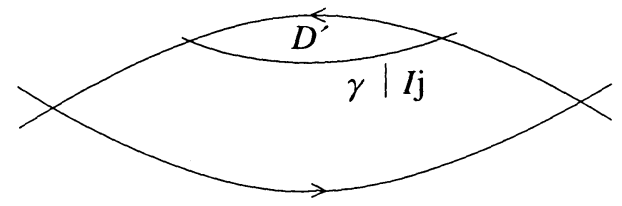

Fig. 11.

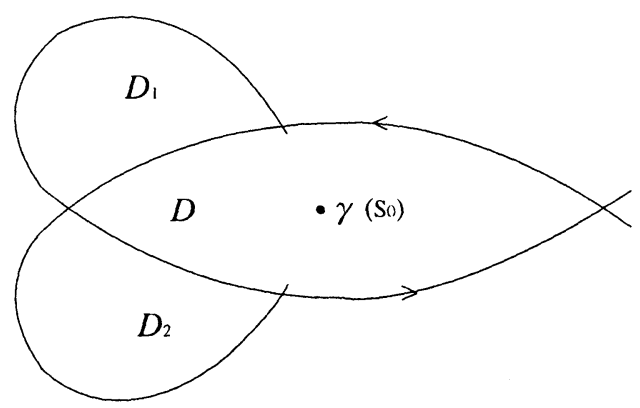

Fig. 12 .

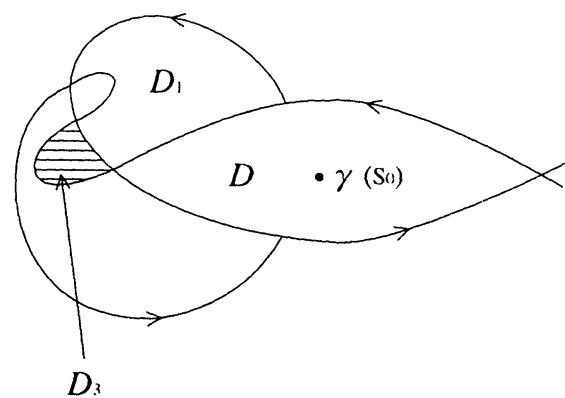

Fig. 13.

Lemma 3.4. Let $\gamma$ be a semisimple closed curve with period $l$. Suppose that $\gamma(a)=\gamma(b)(a<b<a+l)$ such that $\left.\gamma\right|_{[a, b]}$ is not a shell. Then there exist two numbers $a^{\prime}$ and $b^{\prime}\left(a<a^{\prime}<b^{\prime}<b\right)$ satisfying the following properties:

(1) $\gamma\left(a^{\prime}\right)=\gamma\left(b^{\prime}\right)$.

(2) $\left.\gamma\right|_{\left[a, a^{\prime}\right]}$ and $\left.\gamma\right|_{\left[b, b^{\prime}\right]}$ are embeddings.

(3) There is no intersection between $\left.\gamma\right|_{\left(a, a^{\prime}\right)}$ and $\left.\gamma\right|_{\left(b, b^{\prime}\right)}$. 
Proof. Without loss of generality, we may assume that the connecting points satisfy the inequality $a<s_{0}<b<s_{1}<a+l$. Since $\left.\gamma\right|_{(a, b)}$ is not a shell, there is a subinterval $(\alpha, \beta)$ of $(a, b)$ such that $\left.\gamma\right|_{(\alpha, \beta)}$ is a shell. Obviously, $s_{0} \in(\alpha, \beta)$. So $\left.\gamma\right|_{[a, \alpha]}\left(\right.$ resp. $\left.\left.\gamma\right|_{[\beta, b]}\right)$ is a restriction of $\gamma_{2}\left(\right.$ resp. $\left.\gamma_{1}\right)$. Hence $\left.\gamma\right|_{[a, \alpha]}$ and $\left.\gamma\right|_{[\beta, b]}$ are embeddings. Now we consider the point $\gamma\left(a^{\prime}\right)=\gamma\left(b^{\prime}\right) \quad\left(a<a^{\prime}<\alpha, b<b^{\prime}\right.$ $<\beta)$ at which $\left.\gamma\right|_{[a, \alpha]}$ meets $\left.\gamma\right|_{[\beta, \gamma]}$ first time. Then the numbers $a^{\prime}$ and $b^{\prime}$ are the desired ones.

Lemma 3.5. Let $\gamma$ be a semisimple closed curve with period $l$. Suppose that there are two disjoint closed intervals $[a, b]$ and $[c, d](0 \leq a<b<c, d \leq l)$ such that

(1) $\gamma(a)=\gamma(d)(=P), \gamma(b)=\gamma(c)(=Q)$.

(2) $\left.\gamma\right|_{[a, b]}$ and $\left.\gamma\right|_{[c, d]}$ have no intersection on itself and each other.

(3) The internal angles of the domain $D$ bounded by $\left.\gamma\right|_{[a, b]}$ and $\left.\gamma\right|_{[c, d]}$ are greater than $\pi$ at the point $P$ and less than $\pi$ at the point $Q$.

(4) There exists a subinterval $(\alpha, \beta)$ of $(b, c)$ such that $\gamma$ has a shell on $(\alpha, \beta)$ and $\gamma(\alpha)=\gamma(\beta) \notin D$. (Fig. 14)

Then there exists a leaf $D^{\prime}$ as in Fig. 10 contained in $\bar{D}$.

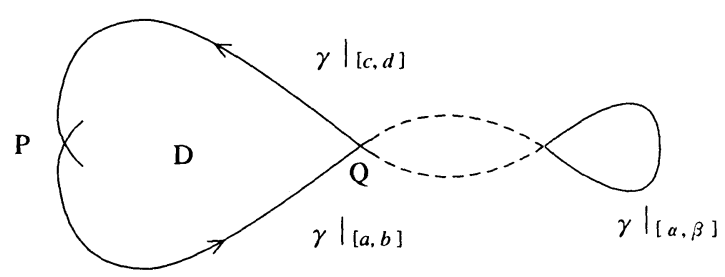

Fig. 14 .

Proof.

Case 1: First we consider the case $\left.\gamma\right|_{(d, a+l)}$ is not contained in $D$. Then there exists a number $d^{\prime}\left(d<d^{\prime}<a+l\right)$ such that $\gamma\left(d^{\prime}\right) \in \partial D$ and $\left.\gamma\right|_{\left(d, d^{\prime}\right)}$ has no self-intersection and contained in $D$. By the property (B), we have $\gamma\left(d^{\prime}\right) \neq P, Q$. If $\gamma\left(d^{\prime}\right)$ lies on the curve $\left.\gamma\right|_{(c, d)}$, then we can take the point $c<c^{\prime}<d$ such that $\gamma\left(d^{\prime}\right)=\gamma\left(c^{\prime}\right)$ and find a positive shell of internal angle $(<\pi)$ on the interval $\left(c^{\prime}, d^{\prime}\right)$. By Theorem 2.1 and the property (B), there exists a negative shell $\left.\gamma\right|_{I}$ on some interval $I$ whose image is contained in the interior of the shell $\left.\gamma\right|_{\left(c^{\prime}, d^{\prime}\right)}$. By the assumption (4) of the lemma, three shells on $(\alpha, \beta),\left(c^{\prime}, d^{\prime}\right)$ and $I$ are inde- 
pendent. But this contradicts the property $(\mathrm{C})$.

So we may assume that $\gamma\left(d^{\prime}\right)$ lies on the curve $\left.\gamma\right|_{(a, b)}$. Then we can take the point $a<a^{\prime}<b$ such that $\gamma\left(a^{\prime}\right)=\gamma\left(d^{\prime}\right)$ and find a leaf as in Fig. 10 on the interval $\left(a, a^{\prime}\right)$ between $\left(d, d^{\prime}\right)$.

Case 2: Next we consider the case $\left.r\right|_{(d, a+l)}$ contained in $D$. If $\left.\gamma\right|_{(d, a+l)}$ is a shell, by the property (B) it must be a positive shell of internal angle $(<\pi)$. By Theorem 2.1 and the property (B), there exists a negative shell $\left.\gamma\right|_{I}$ on some interval $I$ whose image is contained in the interior of the shell $\left.\gamma\right|_{(d, a+l)}$. By the assumption (4) of the lemma, three shells on $(\alpha, \beta),(d, a+l)$ and $I$ are independent. But this contradicts the property (C). Hence $\left.r\right|_{(d, a+l)}$ is not a shell. Then by Lemma 3.4, there exist numbers $d^{\prime}$ and $a^{\prime}\left(a^{\prime}<a<d<d^{\prime}\right)$ such that

(i) $\gamma\left(a^{\prime}\right)=\gamma\left(d^{\prime}\right)\left(=P^{\prime}\right), \gamma(a)=\gamma(d)\left(=Q^{\prime}\right)$

(ii) $\left.\gamma\right|_{\left(a^{\prime}, a\right)}$ and $\left.\gamma\right|_{\left(d, d^{\prime}\right)}$ have no intersection on itself and each other.

(iii) The internal angle at the point $Q^{\prime}$ of the domain $D^{\prime}$ bounded by $\left.\gamma\right|_{\left[a^{\prime}, a\right]}$ and $\left.\gamma\right|_{\left[d, d^{\prime}\right]}$ are less than $\pi$.

If the internal angle at the point $P^{\prime}$ is also less than $\pi$, then we find a leaf as in Fig. 10 on the interval $\left(a^{\prime}, a\right)$ between $\left(d, d^{\prime}\right)$

Finally, we consider the case the internal angle at the point $P^{\prime}$ is greater than $\pi$. Then it is easily seen that two closed intervals $\left[a^{\prime}, a\right]$ and $\left[d, d^{\prime}\right]$ also satisfy the assumptions of Lemma 3.5. Thus we can apply this argument again for the intervals $\left[a^{\prime}, a\right]$ and $\left[d, d^{\prime}\right]$. Since the number of self-intersections of $\gamma$ is finite, we find a leaf as in Fig. 10 by repeating the argument.

Proof of Theorem 3.1. Suppose that there exists a semisimple closed curve which bounds an immersed surface of genus $g>0$. Let $d$ be the minimum number of intersection points of such curves. Since $g \neq 0$, the integer $d$ is positive and we can assume that $\gamma$ represents the one of absolutely minimum self-intersections $d$. By the property (D), we can take an interval $(\alpha, \beta)$ on which $\gamma$ has a negative shell of internal angle $(<\pi)$. If $\left.\gamma\right|_{(\beta, \alpha+l)}$ is also a shell, $\gamma$ has only one self-intersection. But this contradicts the fact that the closed curve which bounds immersed surface has even intersections. (See (D).) Hence $\left.\gamma\right|_{(\beta, \alpha+l)}$ is not a shell and by Lemma 3.4, there exist numbers $a$ and $b(a<\alpha<\beta<b)$ such that

(i) $\gamma(a)=\gamma(b)(=P), \gamma(\alpha)=\gamma(\beta)(=Q)$.

(ii) $\left.\gamma\right|_{(a, \alpha)}$ and $\left.\gamma\right|_{(\beta, b)}$ have no self-intersection on itself and each other.

(iii) The intermal angle at the point $Q$ of the domain $D$ bounded by $\left.\gamma\right|_{(a, \alpha)}$ and $\left.r\right|_{(\beta, b)}$ are less than $\pi$. (See Fig. 15.) 
If the internal angle at the point $P$ is also less than $\pi$, then certain two intersections can be canceled by Lemma 3.3. This contradicts the fact that $\gamma$ has absolutely minimum intersection. Thus the internal angle at the point $P$ is greater than $\pi$. Then it is easily seen that two closed intervals $[a, \alpha]$ and $[\beta, b]$ satisfy all the assumptions of Lemma 3.5. Thus we can find a leaf as in Fig. 10 and cancel two intersections by Lemma 3.3. So this makes also a contradiction. Now we can conclude that there is no semisimple closed curve which bounds immersed surface of genus $g>0$. This proves the theorem.

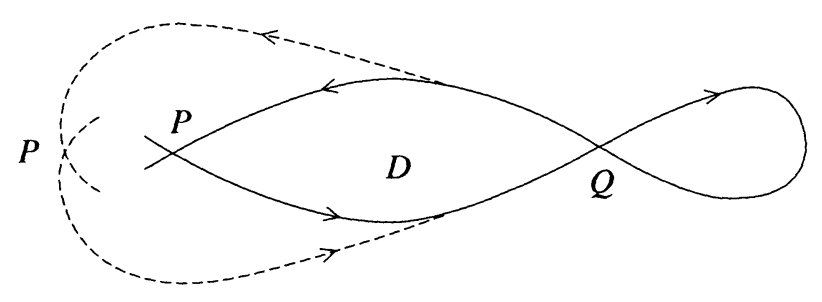

Fig. 15 .

Added in proof. G. Cairns, M. Mclntyre and M. Özdemir (Bull. London Math. Soc., 25 (1993)) also gave a proof of the 6-vertex theorem in Introduction under the additional assumption that the planer curve $\gamma$ is normal (i.e. all their intersections are at most double points and transversal). Their approach is quite different from ours.

\section{REFERENCES}

[COT] G. Cairns, M. Özdemir and E. H. Tjaden, A counterexample to a conjecture of U. Pinkall, Topology, 31 (1992), 557-558.

[J] S. B. Jackson, Vertices of plane curves, Bull. Amer. Math. Soc., 50 (1944), $564-578$.

[KB] L. H. Kauffiman and T. F. Banchoff, Immersions and Mod-2 quadratic forms, Amer. Math. Monthly, 84 (1977), 168-185.

[P] U. Pinkall, On the four-vertex theorem, Aequat, Math., 34 (1987), 221-230.

[W] H. Whitney, On regular closed curves in the plane, Comp. Math., 4 (1937), $276-284$

Department of Mathematics

Faculty of Science

Osaka University

Toyonaka, Osaka 560, Japan. 